\title{
Computational and experimental characterization of critical amino acidic residues in the BCR-ABL kinase domain explaining TKIS resistance in patients with chronic myeloid leukemia
}

\author{
C. Romano, P. Buffa, A. Pandini, M. Massimino, E. Tirrò, L. Manzella, F. Fraternali, P. Vigneri ${ }^{凶}$ \\ Department of Clinical and Molecular Bio-Medicine, University of Catania, Catania, Italy
}

\section{Motivations}

Suppression of BCR-ABL1 catalytic activity by the Tyrosine Kinase Inhibitor (TKI) Imatinib Mesylate (IM) has dramatically improved the natural history of Chronic Myeloid Leukemia (CML) ushering the era of molecular targeted therapy. Despite the unparalleled results achieved by IM, 30\% of CML patients become resistant to the compound, mostly because of point mutations that interfere with drug binding. Thus, the need for a structural characterization of the interactions between the BCR-ABL kinase domain and different TKIs, that will define the mechanisms allowing BCR-ABL mutants to avoid kinase inhibition by most of these drugs.

\section{Methods}

Point mutations affecting BCR-ABL Kinase domain are not randomly distributed in the BCR$A B L$ kinase sequence. Indeed, only one (T315) of five residues critical for IM interaction is the object of amino acidic substitutions in IM-resistant patients. We have computationally investigated why these five amino acids (E286, T315, M318, 1360, D381) are critical for IM binding. Two of them (T315 and M318) are maintained in binding the second generation (2G) TKI Dasatinib (DAS), while E286, M318, 1360 and D381 are required for the interaction with the third generation (3G) inhibitor Ponatinib (PON). We generated tagged-BCR$A B L$ constructs displaying conservative or nonconservative mutations in each of these amino acidic residues. Every BCR-ABL mutant was lentivirally transduced in Ba/F3 cells and evaluated for expression, catalytic activity, transforming potential and response to TKI treatment. We then performed 50ns Molecular Dynamics (MD) simulations of the mutants showing catalytic activity in a simulated aqueous environment using the GROMACS package with AMBER force field (ffamber99sb), obtaining 50ns of data collection for each system. We also performed MD simulations of $A B L K D$ in complex with the two approved therapeutic inhibitors, IM and DAS, and with PON. These simulations were aimed at highlighting if and how the mutations influenced inhibitor binding.

\section{Results}

Combining computational and biological approaches we demonstrate that, with the exception of T315, the four remaining amino acids critical for TKI interaction are pivotal to preserve both BCR-ABL kinase activity and oncogenic potential. The conservative mutation I360T was the only substitution capable of maintaining BCR-ABL kinase activity and transforming potential. However, BCR-ABLI360T remained sensitive to TKI treatment. We also employed Molecular Dynamics (MD) simulations to probe molecular motions at an atomic scale and investigate the dynamical features of the mutants that could not be extracted from static structures. The I360T mutation clearly induces a displacement of the C-helix, taking away E286 from the catalytic pocket. This residue is critical for $\mathrm{IM}$ binding. To establish if this is a disadvantageous condition for IM stability, we docked this drug inside the pocket of the structure performing further 50ns of MD simulation. Preliminary results suggest that not only the C-helix moves back towards the catalytic pocket (maybe under the electrostatic field generated by the drug) but also that IM restores three of the five original h-bond interactions guarantying IM binding. This result seems to be in agreement with our experimental data showing that BCRABLI360T is inhibited by IM. Analysing the interactions between the BCR-ABL kinase domain and different TKIs, our data confirm the efficacy of $3 G$ inhibitor Ponatinib on CML patients failing IM and $2 G$ TKIs, since the interaction with the T315 residue (the only one among five susceptible of mutation) is lost. These findings may be extended to further protein kinases involved in solid and hematologic malignancies, thus contributing to the design of additional TKIs. 